\title{
Analysis of open innovation communities from the perspective of social network analysis
}

\author{
M.R. Martínez-Torres* \\ Department of Business Administration and Management, University of Seville, Seville, Spain
}

\begin{abstract}
Open innovation is an emergent paradigm by which organisations make use of their internal and external resources to perform their innovation processes. The growth of information and communication technologies has facilitated the spread of online open innovation communities, where users can share ideas as well as comment on and evaluate ideas posted by other community members. In this work, the behaviour of community members is analysed from the perspective of social network analysis. The final aim is twofold: first, to measure to what extent the different forms of participation are correlated to each other; and, second, how the collective intelligence evaluation schemes can be useful to identify those users posting ideas which are potentially applicable for the organisation. Obtained results can help community managers and organisations to improve the efficiency of the evaluation process when hundreds or thousands of ideas are shared through the online community.
\end{abstract}

Keywords: open innovation communities; online communities; social network analysis; zero inflated problems

\section{Introduction}

Open innovation refers to the use of external resources and partners to improve the innovation capacity of the organisation, and relies on the idea that invention and innovation can emerge both inside and outside the company's walls (Banerjee, Friedrich, and Morell 2010; Chesbrough, Vanhaverbeke, and West 2006). Consequently, open innovation processes involve a wide range of internal and external technology sources, and a wide range of internal and external technology commercialisation channels (Galbraith and McAdam 2011). Different from the traditional innovation model, this paradigm also assumes that the risks derived from opening the innovation, such as the access to valuable information by competitors or the loss of control over the innovation process, can be compensated by a richer number of innovative ideas. The active integration of customers contributes to reduce uncertainty about the market. This integration means that customers become an active designer in the process of innovation.

\footnotetext{
*Email: rmtorres@us.es
} 
Open innovation communities have emerged as popular mechanisms to integrate customers as part of the innovation process. This trend is explained because the Internet offers global accessibility and facilitates communication and interaction between contributors at a comparatively low cost. The most viable approach for using the crowd as a resource is through open innovation platforms on the Internet, which have exponentially grow during the last years (Jeppesen and Lakhani 2010; Mahr and Lievens 2012). Through these virtual environments, users or experts can share ideas with the rest of the community, which can be geographically distributed all over the world. In this sense, they are connected with communities of practice, which are communities of users organised around circumscribed sets of activities, and their members develop their own routines, formal and informal rules, and practices evolve as a result of learning (Wenger 1998).

Although open innovation communities constitute a form of virtual community based on crowdsourcing, they exhibit important differences with respect to other type of communities based on mass collaboration like open source software communities or collaborative research communities. While in open innovation communities the focus is on the firm or the product, and users are usually allowed to participate by posting, commenting or evaluating ideas, the mass collaboration takes the form of self-organising bottom-up driven movements, where the idea is the carrier and focus of the organisation (Elmquist, Fredberg, and Ollila 2009; Martínez-Torres and Díaz-Fernandez, forthcoming). This is the case of open source communities, which exhibit a clear onion-like structure, with a central core of highly active individuals, surrounded by other layers of progressively less-active individuals (Martínez-Torres et al. 2012). Open source communities do not implement a collective evaluation system. Posting innovations requires a certain level of expertise and modifications are decided by a reduced group of experts that constitutes the core of the community. On the contrary, open innovation communities are open to all registered customers and users. They do not require a high level of expertise and users are free to comment on and score other users' ideas. The final decision about the suitability of ideas is carried out by the company, considering their own experts' opinions and their innovation strategic policies.

One of the most important challenges of the open innovation paradigm is its effectiveness in terms of performance. Prior studies have found a nonlinear relationship between open innovation and performance (Laursen and Salter 2006). The main problem is that the huge amount of generated information saturates a company's absorptive capacity (Clausen 2013). Posted ideas should be evaluated one by one by the innovation department, even with the help of experts of the organisation in some specific topics. Ideas' evaluation consists of reading the idea, assessing its applicability attending to the strategic innovation policies of the organisation and planning their possible implementation in case they were finally accepted.

Some authors have tried to solve this problem by introducing a special group of users, called lead users (von Hippel 1986, 1988). They are characterised because they can foresee innovations much earlier than the rest of the users. Lead users have also the ability to develop a fully functional solution for their needs (Mahr and Lievens 2012; Morrison, Roberts, and Midgley 2004). Therefore, they can be used to foresee future trends. According to several authors (Morrison, Roberts, and Midgley 2004; Urban and von Hippel 1988), lead users are more active in the community than the rest of the users of the community. That is, this specific group of users can be identified by their characteristics of participation inside the community.

This paper follows this approach and analyses the patterns of behaviour of the community users by modelling the community as a social network, where nodes represent the community users and arcs represent their interactions. Social network analysis has been frequently used to analyse the key users in open source communities (Toral, Martínez-Torres, and Barrero 2010) or the key players in the mobile ecosystem (Basole 2009). Both of them correspond to a micro structural 
analysis based on the topological properties of nodes. In the case of open innovation communities there is a clear partition consisting of those nodes whose ideas have been finally implemented by the company, as opposed to the rest of the users. Information about ideas implemented by the company is publicly shown, as a general recognition to those users who post interesting ideas and to encourage the participation of the rest of the users. The main contribution of this paper consists of obtaining the features of participation which are more relevant to identify innovative users, defined as those users whose ideas are finally implemented by the company. The concept of influencer is specific to those communities open to a wide audience, like open innovation communities or consumer communities. They do not need a certain level of expertise or to become an expert through a learning process, as it is typical in more specialised virtual communities, where the profile of brokers of knowledge is more extended (Sowe, Stamelos, and Angelis 2006). Additionally, their identification can save a lot of effort and human resources in the process of evaluating ideas with potential applicability.

The rest of the paper is organised as follows: the following section details the research framework. Section 3 introduces the case study and the definition of variables. Section 4 explains the proposed methodology and Section 5 shows the obtained results, which are discussed in Section 6 as well as the main implications and limitations of this work. Finally, the paper is concluded in Section 7.

\section{Research framework}

Open innovation communities are organised around the possible innovations that could be implemented on the products and services of an organisation, and they have proliferated from the emergence of Web 2.0 sites. They are different from other online communities that work as digital repositories of information where users post information at will, and contributions do not respond to any specific request for information (Martínez-Torres and Díaz-Fernandez, forthcoming).

Open innovation communities allow each registered user to participate in sharing ideas, posting comments or evaluating ideas posted by other users. Previous works show that users of an open innovation community tend to participate in all the allowed modalities through the open innovation websites (Toral, Martínez Torres, and Di Gangi 2011). The most typical form of participation consists of posting ideas. However, community users can also refine, improve or criticise a previously posted idea by posting comments associated to this idea. According to previous research about participation in open innovation communities, the user's motivation to post ideas or comments depends on factors like learning, cooperation and entertainment (Antikainen, Mäkipää, and Ahonen 2010). Following the social learning theory of Wenger (1998), learning implies participation in a social environment, as knowledge is acquired in the context in which it is generated. That means that the users' interactions through shared ideas and comments promote learning from the community. Cooperation is achieved with the flow of resources in the community, which facilitates the generation of new ideas (Faraj, Jarvenpaa, and Majchrzak 2011). Finally, entertainment is stimulated when users feel engaged with the community, which in turn is a consequence of the successful interactions with other users commenting or scoring ideas. As a result, activity of users is encouraged when the posted ideas or comments stimulate the interest of other users, or generate a debate or discussion. Sometimes the company participates in the discussion through a specific user to clarify some aspects of the posted ideas.

The modality of participation scoring other users' ideas provides a measurement about user reputation. The received feedback in the form of a rating gives an idea of the confidence one can have on the ideas posted by a specific user (Pujol, Sangüesa, and Delgado 2002). Companies 
make use of this kind of community-based idea evaluation to facilitate the process of identifying the best ideas (Berg-Jensen et al. 2010). Using the scoring system, the community performs a collective judgment task improving the knowledge transmission between the community and the company (Blohm et al. 2011; Leimeister 2010). Furthermore, selecting the most popular ideas has the advantage of transmitting the message that the company is committed to users' necessities. However, the disadvantage is that this system requires the explicit and frequent involvement of users that issue ratings, and the fact that most popular ideas are not always aligned with the company innovation strategic policies.

This paper analyses the participation of community users by modelling their interactions as social networks. Using those users whose posted ideas were finally implemented as the dependent variable, this paper demonstrates that community-based evaluation fails in finding those ideas that will finally be implemented by the company. Moreover, this paper identifies the features or variables which are more appropriate for the identification of innovative users.

\section{Case study and definition of variables}

Dell IdeaStorm is a user innovation community where end users freely reveal innovative ideas with the community members and Dell to improve its products and services (Di Gangi and Wasko 2009). This website represents a new way to listen to customers on how to build the best products and services. Through IdeaStorm, customers can post their ideas about existing and/or new Dell products, services and operations. They have also the option of commenting other users' posted ideas, suggesting improvements or criticising them. As a result, a debate is generated around those posted ideas which attract the interest of the community. Moreover, users have the option of scoring ideas following a collective evaluation system, through which users can promote or demote ideas. Promoting an idea adds 10 points to its current rating, while demoting an idea subtracts 10 points. Users are free to decide which ideas they want to comment on, promote or demote, and they can also choose to do any of the three possible actions. Users have to register with an alias to be allowed to participate in the community. This alias is unique and identifies users whenever they post an idea, a comment or a rating. Each user can participate as long as they are registered. From the researcher point of view, one of the most attractive characteristics of open innovation communities is the fact that historic information is publicly available.

A total of 1482 ideas shared were collected and processed. For each idea, the information listed in Table 1 was extracted.

Table 1. Data extracted for each shared idea.

Title of posted idea
Alias of the user that posted the ideas
Comments
$\quad$ Number of comments received
$\circ$ Alias of users commenting on the idea
Promotions
$\circ$ Number of promotions received
$\circ$ Alias of users promoting the idea
De Number of demotions received
$\circ$ Alias of users demoting the idea


The community can be modelled as a social network, considering users as nodes and arcs as interactions among users. Taking into account the mentioned modalities of participation, three different social networks can be extracted: a comments network, a promotions network and a demotions network.

\section{Comments network}

The comments network was built by processing the authors of comments generated by the considered 1482 shared ideas. The total size of this network is 1381, which includes those users who have contributed to the community posting ideas or comments. The resulting network is a valued directed graph, which means that several interactions between the same users can take place, and that the arcs are directed from users commenting on ideas to the user who originally posted the idea. Figures 1 and 2 display the comments network. The area of nodes has been drawn proportional to their in-degree value in the case of Figure 1, and to their out-degree value in the case of Figure 2.

The 1381 nodes of the network include the 1153 users that have at least posted one idea plus the 208 users who have only participated by commenting on ideas without actually sending an original one.

Nodes with a higher in-degree value in Figure 1 are those users whose posted ideas and received more comments from the rest of the community, that is, those users whose ideas aroused more discussion and debate within the community. Out of the 1381 community of users, 808 have an in-degree value of zero, including the 208 users that never posted an idea and those users that posted an idea but never received any comment. Only 315 users that posted ideas received one or more comments.

Similarly, nodes with a larger size in Figure 2 are the most active users commenting on shared ideas. Collected data reveal that only 408 users posted at least one comment, while the rest of the 953 users did not participate in the community by commenting on ideas.

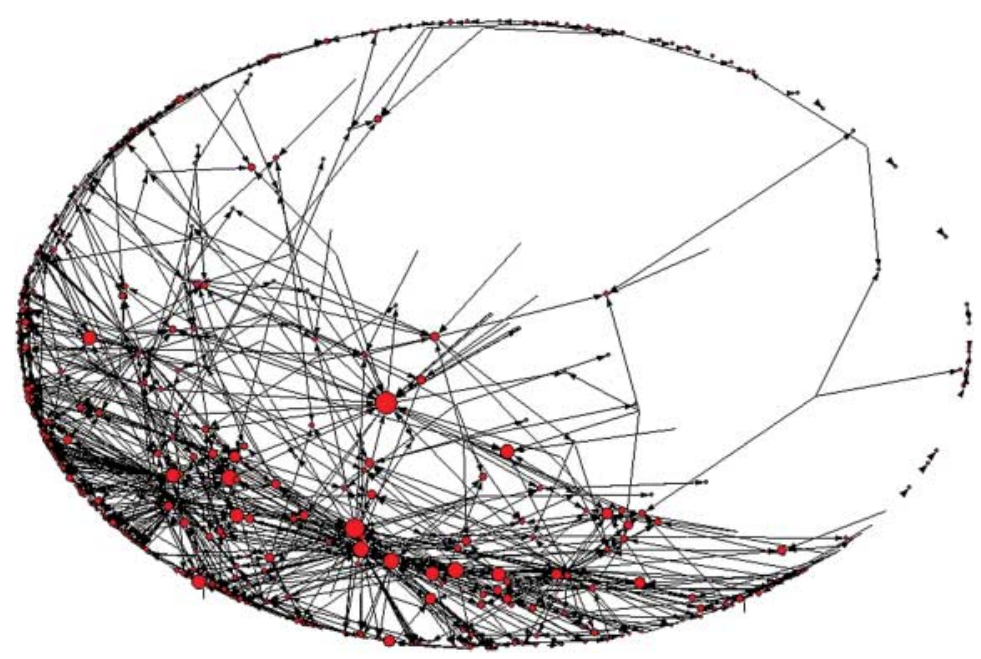

Figure 1. In-degree values of the comments network. 


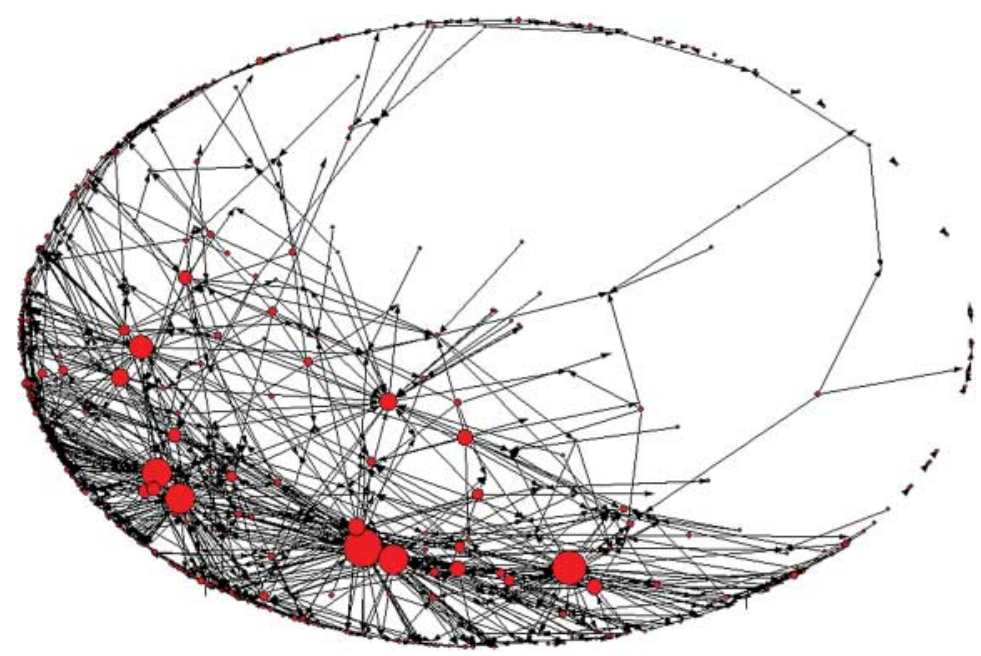

Figure 2. Out-degree values of the comments network.

In summary, open innovation communities follow up an inequality participation scheme similar to other online communities. This inequality participation means that a majority of contributions are posted by a small fraction of the community (Toral, Martínez-Torres, and Barrero 2010).

\section{Promotions network}

The promotions network is built considering users as nodes and arcs as the links between users promoting an idea and the author who originally posted this idea. Figures 3 and 4 show the promotions network, emphasising with a larger area those nodes with a higher in and out degree, respectively. In this case, the network size is 2151. Again, it can be distinguished between the 1153 users who have posted at least one idea, and the 998 users who have promoted ideas but have never posted an idea.

Figure 3 clearly illustrates that shared ideas receive more promotions than comments in comparison to the corresponding comments network of Figure 1. This could be explained because it is much easier rating an idea than commenting on it. Rating ideas do not require any justification, while commenting on an idea requires previous knowledge about the addressed subject.

Figure 4 shows the activity of the members of the community when promoting ideas. Although nodes appear overlapped, the same scale has been maintained when representing the area of nodes for comparison purposes with previous networks. It can be noticed that some specific users are quite prolific sending promotions (there are 5 users with an out-degree higher than 500). Most users have promoted at least one idea.

\section{Demotions network}

The demotions network is built in a similar way to the previous network, but using demotions instead of promotions. The network size is 1459 (that is, 1153 users have posted at least one idea and 306 users have demoted ideas but have never posted an idea). The meaning of the demotions network is the same as the promotion network, but using the idea of demotion instead of promotion. 


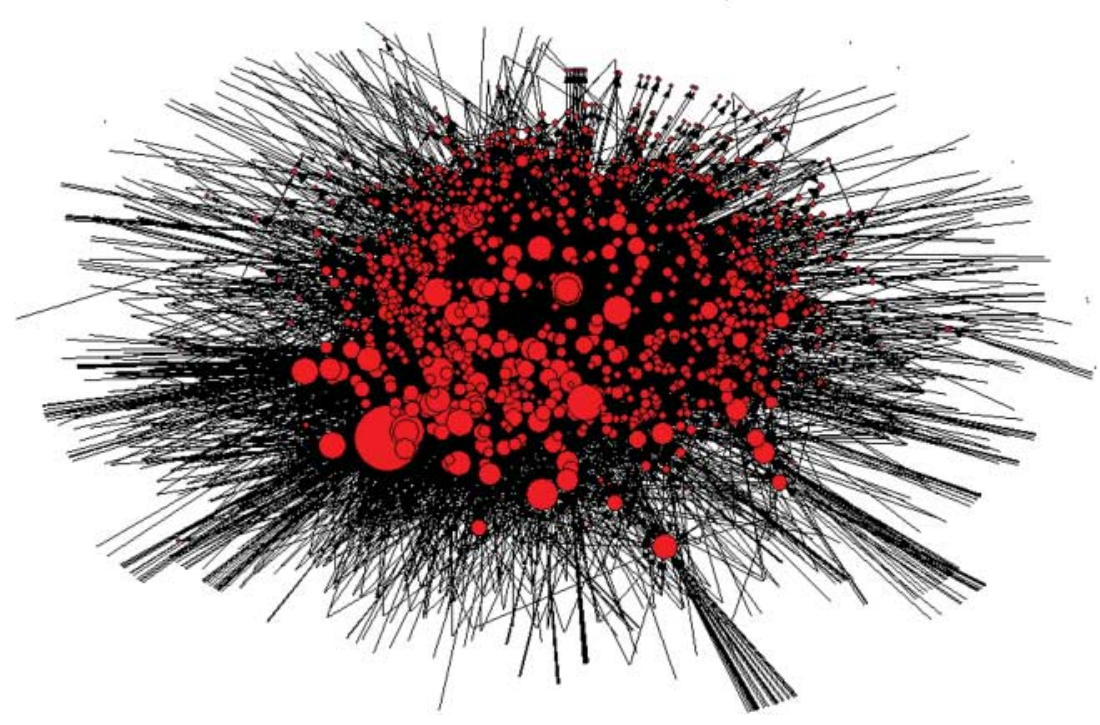

Figure 3. In-degree values of the promotions network.

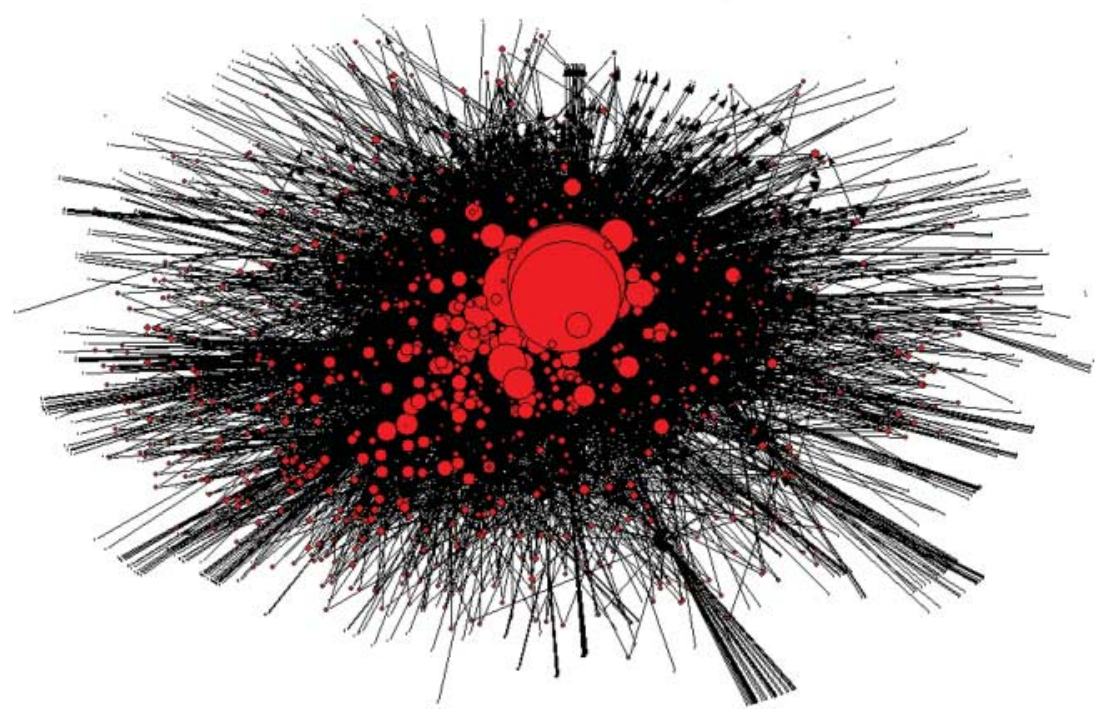

Figure 4. Out-degree values of the promotions network.

Figures 5 and 6 displays the demotions network, the size of the nodes being proportional to their in and out degrees. As in the corresponding promotions networks, Figure 5 confirms that shared ideas generate more activity through scoring than through comments. Figure 6 illustrates the presence of very active 'demoter' users with a huge out-degree value.

The degree distributions of the three considered networks point out that the three of them exhibit features of scale-free networks rather than random networks. 


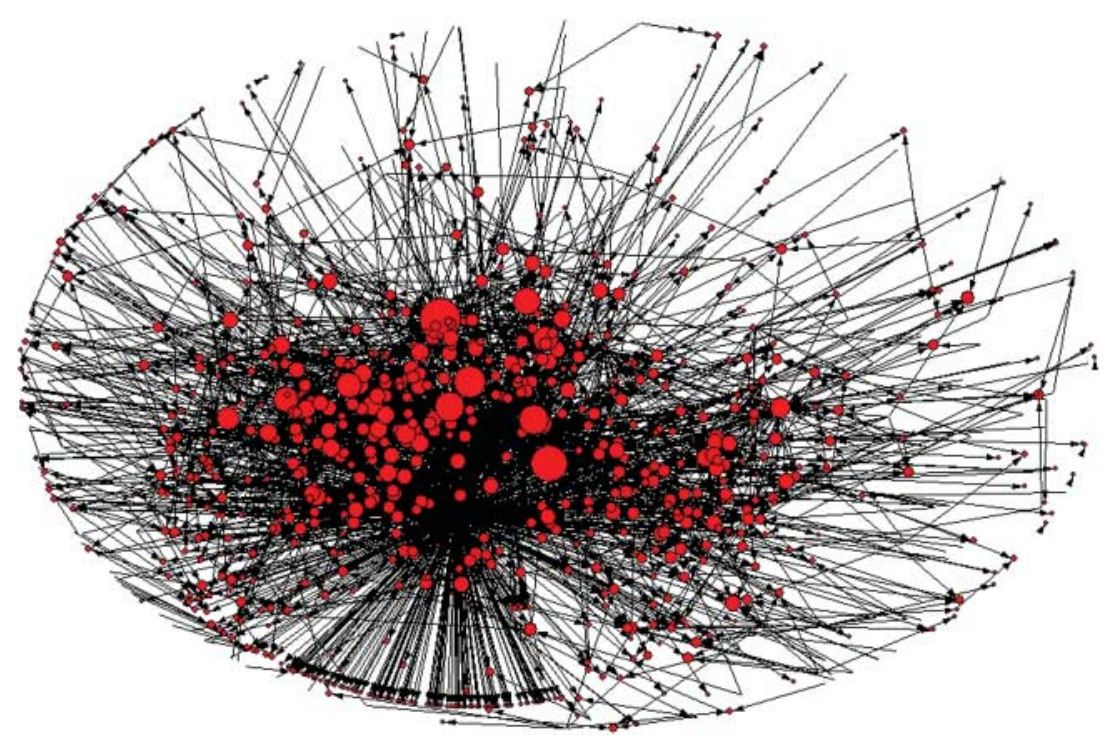

Figure 5. In-degree values of the demotions network.

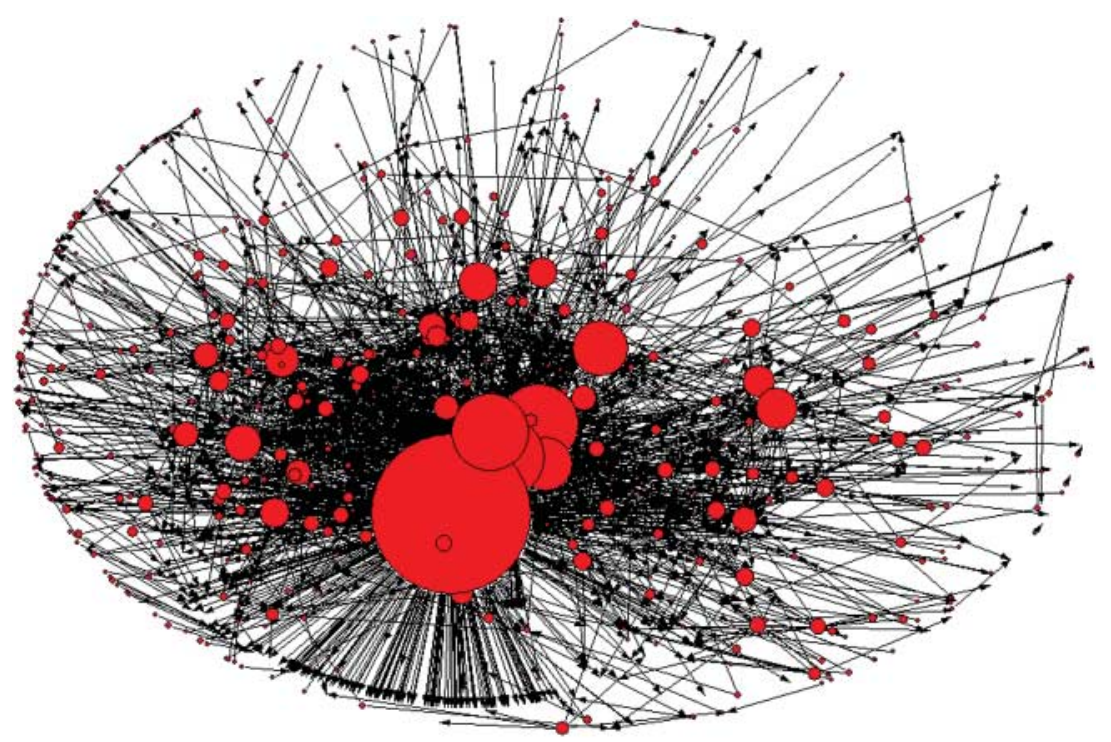

Figure 6. Out-degree values of the demotions network.

Table 2 shows the clustering coefficient for each of the three considered networks and for the equivalent Erdos-Renyi networks. It can be noticed that equivalent Erdos-Renyi networks have a clear lower value for the clustering coefficient than the original networks. This table also shows how the degree distributions of the three considered networks can be better fitted by a power law distribution (Figure 7). The $\alpha$ coefficient was estimated according to the goodness-of-fit-based method described in Clauset, Shalizi, and Newman (2009). The Kolmogorov-Smirnov test $D$ value for both of them are below the critical value given by $1.63 / \mathrm{N}^{0.5}$. 
Table 2. Comparison of the three obtained networks with Erdos-Renyi and scale-free networks.

\begin{tabular}{lcccccc}
\hline & & \multicolumn{2}{c}{ Erdos-Renyi } & & \multicolumn{2}{c}{ Scale-free } \\
\cline { 3 - 4 } Networks & CC & $P$ & CC & & Alpha & $D$ \\
\hline Comments & 0.0171 & $6.16 \mathrm{e}-4^{* * *}$ & 0.00141 & & 2.06 & 0.039 \\
Promotions & 0.0163 & $6.17 \mathrm{e}-4^{* * *}$ & 0.00232 & & 2.46 & 0.018 \\
Demotions & 0.0067 & $1.40 \mathrm{e}-3^{* * *}$ & 0.00069 & & 2.11 & 0.034 \\
\hline
\end{tabular}

${ }^{* * *} p<0.001$

(a)

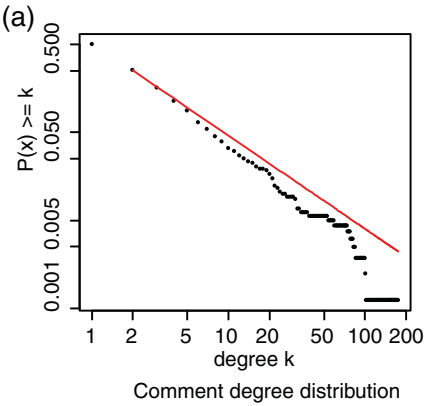

(b) 8

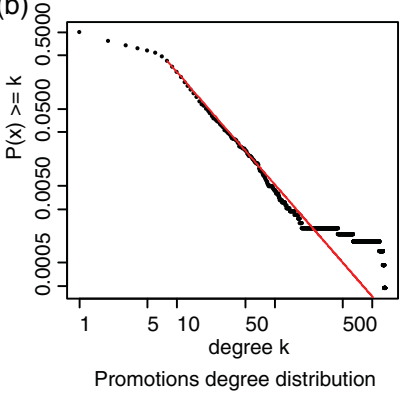

(c)

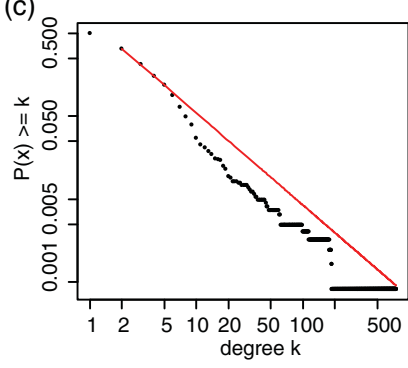

Demotions degree distribution

Figure 7. Degree distributions and power law fit.

\section{Methodology}

This section details the methodology used to perform the proposed study. Three basic steps have been taken into account. The first one consists of designing and programming a crawler to extract the data from the open innovation website. The second one consists of modelling the community as a social network and extracting its topological features. The final step describes the regression analysis used to find the variables which characterise innovative users.

\section{Data extraction}

A crawler has been designed and programmed to extract the data from the IdeaStorm open innovation website. These websites are typically structured through shared ideas. For each one of them, the author (a registered user identified by an alias), the received comments and the ratings are shown. The designed crawler was designed to access the source code of the webpage and to extract the alias of community users as well as their interactions, as shown in Figure 8.

Basically, the crawler performs a double processing of webpages, as shown in the two inner loops of Figure 8. In the first loop, those webpages which contain the shared ideas are accessed and processed to obtain the final list of users. These users are identified by an alias that they choose when registering in the community. The second loop obtains the interactions among users, extracting and processing those users that have posted comments or ratings to shared ideas. The main advantage of using a specific crawler instead of a general one is that the content can be filtered and processed to obtain the desired elements (Martínez-Torres 2012). 


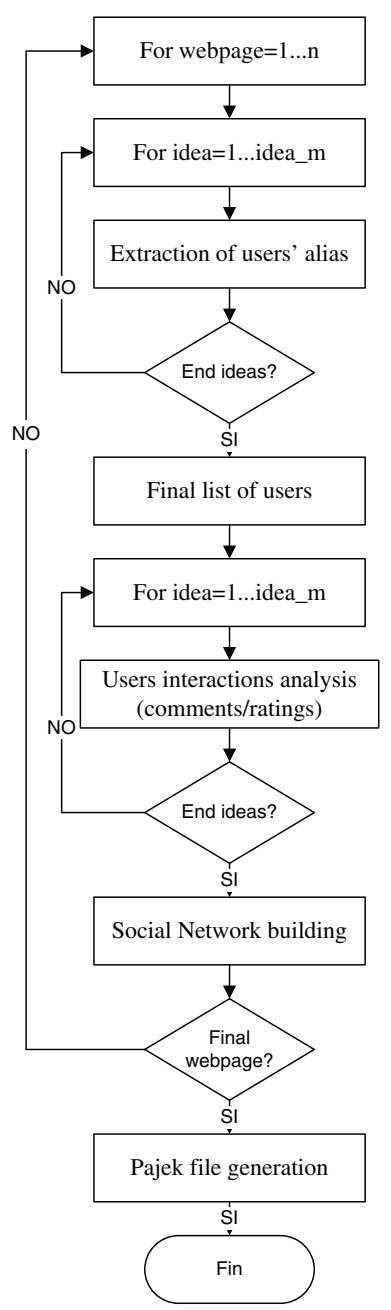

Figure 8. Flow diagram of the designed crawler for extracting users and their interactions in open innovation communities.

\section{Social network analysis}

A social network is defined as a set of nodes connected by ties, and it is primarily concerned with the interconnections between individuals, rather than being focused on their attributes or behaviours (Wellman 1983). This is the only available information through online communities, where users are identified by an alias and their activity is recorded in the form of messages, comments or ratings. When building the social network, it has been considered that a user sending a comment to a shared idea is linked not only to the author of the idea, but to all the community users who previously submitted comments on the same idea (Toral, Martínez-Torres, and Barrero 2010). The reason is that it is more cognitively complex to reply to a threaded discussion than to a single message, because the flow of earlier messages should be considered to post a coherent new comment (Knock 2001). 
Once the network is built, social network analysis techniques provide a powerful means for describing and analysing individuals within a community structure. One of the most representative features of each node is its degree, which refers to the number of lines incident with it (Toral, Martínez-Torres, and Barrero 2009). In the case of directed networks, the in-degree of a node refers to the number of arcs it receives, while the out-degree refers to the number of arcs it sends. The out-degree of a given user is an indicator of his or her activity in the different modalities of participation allowed by the innovation website, while the in-degree represents the popularity or reputation of each user (Lin and Kao 2010). This feature associated to the community of users will be correlated to characterise their behaviour.

\section{Regression analysis}

A regression analysis will be used to find the variables that characterise the behaviour of innovative users. The dependent variable is the condition of being or not being an innovative user, which can be easily extracted from open innovation websites, since they usually provide informations about those ideas that have been finally adopted. The main problem with this dependent variable is that only a small fraction of shared ideas are finally adopted by the company. Consequently, the dependent variable contains a high number of zeros (which is the value assigned to the noninnovative users) and a low rate of ones (which is the value assigned to innovators). This kind of problems where the dependent variable contains a disproportionally high number of zeros are known as zero inflated problems, and they can lead to biased/inconsistent parameter estimates, inflated standard errors and invalid inferences (Jang 2005; Lee et al. 2006). Sometimes, these problems can be addressed using zero inflated models, which consider a mixture of models to deal with the excess zeros (Lambert 1992). The justification for splitting the distribution into two pieces is because zero values can be structural zeros, which are inevitable, or sampling zeros, which occur by chance. However, this is not the case of open innovation communities, where all the zeros are sampling zeros. The reason is that whenever a user is sharing an idea, he has the expectation that his idea is going to be adopted. The fact of implementing or not implementing the idea relies on the company and its experts. Therefore, there are no structural zeros and no reason for considering a mixture of models. The alterative consists of considering generalised linear modelling with Poisson distribution, because the response is discrete and includes zeros responses. But often generalised linear modelling with Poisson distribution has problems with over-dispersion (Hinde and Demetrio 1998). The model with negative binomial distribution is an alternative way to fix the over-dispersion problem in Poisson distribution (Hinde and Demetrio 1998), as the variance and mean are not assumed to be equal. This is the chosen regression model for this study.

\section{Results}

In- and out-degrees of the comments, promotions and demotions networks are the independent variables that have been considered in this study. They measure several properties of users as part of the community. The three out-degree values refer to the activity of the users in the three modalities offered by the IdeaStorm website (Out_Comm, Out_Prom, Out_Dem). The in-degree of the comments network (In_Comm) measures the users' popularity. In this case, users are visited whenever other users read their posted idea and decide to post a comment. A high number of comments received means the shared ideas are attracting the interest of the community. The scores received through promotions and demotions constitute a measure about the good or bad 
Table 3. Correlation matrix of defined variables.

\begin{tabular}{llllllll}
\hline & In_Comm & Out_Comm & In_Prom & Out_Prom & In_Dem & Out_Dem & Innov \\
\hline In_Comm & 1.000 & $0.363^{* *}$ & $0.274^{* *}$ & $0.190^{* *}$ & $0.196^{* *}$ & $0.146^{* *}$ & $0.204^{* *}$ \\
Out_Comm & $0.363^{* *}$ & 1.000 & $0.315^{* *}$ & $0.461^{* *}$ & $0.144^{* *}$ & $0.434^{* *}$ & $0.073^{*}$ \\
In_Prom & $0.274^{* *}$ & $0.315^{* *}$ & 1.000 & $0.402^{* *}$ & $-0.079^{* *}$ & $0.276^{* *}$ & $-0.059^{*}$ \\
Out_Prom & $0.190^{* *}$ & $0.461^{* *}$ & $0.402^{* *}$ & 1.000 & $0.170^{* *}$ & $0.588^{* *}$ & 0.057 \\
In_Dem & $0.196^{* *}$ & $0.144^{* *}$ & $-0.079^{* *}$ & $0.170^{* *}$ & 1.000 & $0.134^{* *}$ & 0.008 \\
Out_Dem & $0.146^{* *}$ & $0.434^{* *}$ & $0.276^{* *}$ & $0.588^{* *}$ & $0.134^{* *}$ & 1.000 & 0.054 \\
Innov & $0.204^{* *}$ & $0.073^{*}$ & $-0.059^{*}$ & 0.057 & 0.008 & 0.054 & 1.000 \\
\hline
\end{tabular}

${ }^{*}$ Correlation is significant at the 0.05 level (two-tailed).

${ }^{* *}$ Correlation is significant at the 0.01 level (two-tailed).

reputation of a given user (In_Prom, In_Dem), respectively. As it was shown in the comments, promotions and demotions networks of Figures 1-6, the activity of users commenting on ideas is quite different from their activity rating ideas.

The dependent variable is given by those users whose ideas were finally implemented by Dell (Innov). Table 3 shows the Spearman's rank-order correlations between all these variables for the 1153 users that posted at least one idea. The Spearman's rank-order correlation is the nonparametric version of the Pearson product-moment correlation, and measures the strength of the association between ranked variables, that is, how closely several sets of rankings agree with each other (Rencher 2002).

When analysing the partitions related to the in-degree values, a positive but low correlation can be noticed between comments and scoring (both positive and negative) received. That means that ideas that give rise to more debate in the community are not always the best evaluated by the community. Correlation between promotions and demotions received is almost zero, which means that the community is able to clearly distinguish interesting ideas and to discriminate them from those with no potential of applicability. Therefore, it can be said that collective intelligence is working in the sense of discriminating ideas.

Regarding the out-degree values, Table 3 shows that those active users posting comments are also active promoting and demoting ideas. This means users tend to be active in all the modalities of participation offered by the open innovation website. The highest correlation coefficient value is obtained for promotions and demotions, which result also supports the fact that a higher percentage of users prefer to participate by scoring ideas rather than commenting on them.

The last column of Table 3 shows the correlation of innovators, defined as users posting ideas which have been implemented by Dell, with the rest of the variables charactering the participative behaviour of the community of users. It can be observed that the highest correlation coefficient is obtained with the number of comments received. However, the correlation with the number of promotions received or the activity of users is low and non-significant in some cases. A possible interpretation could be that the evaluation system based on collective intelligence is able to discriminate good ideas from poor ones, but in many occasions these well-evaluated ideas have scarce applicability or are far from the company innovation strategies. In the case of the IdeasStorm website, ideas are scored by users without any previous information from the organisation; so users follow their own feelings when scoring them. This result points out that there is a lack of agreement or alignment between the interest of users and the interest of the company.

A negative binomial regression has been applied next to identify the key determinants of the condition of being an innovator. The results of the estimation are shown in Table 4. 
Table 4. Negative binomial regression results.

\begin{tabular}{lcc}
\hline & \multicolumn{2}{c}{ Model estimation } \\
\cline { 2 - 3 } & Coefficient & $p$-Value \\
\hline Constant & $-3.416^{* * *}$ & $<2 \mathrm{e}-16$ \\
In_Comm & $0.227^{* * *}$ & $2.38 \mathrm{e}-12$ \\
Out_Comm & $0.087^{*}$ & 0.019 \\
In_Prom & $0.071^{* * *}$ & 0.000 \\
Out_Prom & -0.007 & 0.264 \\
In_Dem & -0.019 & 0.629 \\
Out_Dem & 0.007 & 0.825 \\
Log-likelihood at maximum & -298.120 & \\
Wald chi-squared & $753.100^{* * *}$ & \\
Akaike information criteria & 316.119 & \\
Bayesian information criteria & 361.571 & \\
McFadden pseudo $R^{2}$ & 0.162 & \\
Maximum-likelihood pseudo $R^{2}$ & 0.048 & \\
Cragg and Uhler's pseudo $R^{2}$ & 0.183 & \\
\hline$* p<0.05$. & & \\
$* * * p<0.001$. & & \\
&
\end{tabular}

The negative binomial regression model predicting innovators is significant and reveals that the number of comments received is the main predictor of the condition of being an innovator. The number of promotions received and the number of comments sent have also positive and significant coefficients, but with a much lower coefficient value. The other three variables related to the promotions and demotions network are not significant. These results highlight the importance of comments in open innovation communities. Popularity measured in terms of comments received is the main antecedent of being an innovator, even ahead of the number of promotions received. Furthermore, the most useful indicator of activity of users for identifying innovators is the activity of commenting on other users' ideas.

\section{Discussion}

This paper analyses the participation features of users in open innovation communities and considers how these participation characteristics can help to identify the important subset of users proposing ideas that are potentially applicable. The analysis of patterns of behaviour in virtual communities has been previously used to detect special group of users such as brokers of knowledge (Sowe, Stamelos, and Angelis 2006) or core team members (Martínez-Torres et al. 2010). The reason is that virtual communities are open to users and contributors all over the world, and they are only identified through an alias. Therefore, the only available personal information is the activity within the community. This information is stored and can be analysed for the whole community as a social network (Pujol, Sangüesa, and Delgado 2002). Therefore, open innovation communities can be analysed as social network where each individual is characterised by his behaviour with respect to the rest of the community. The main distinctive characteristic of open innovation communities is that posted ideas are independently evaluated by the company (status associated to each shared idea) and by the community in the form of a scoring also assigned to 
each idea. It is expected that collective intelligence applied to idea evaluation can help the organisation when selecting the best ideas to be finally implemented. However, obtained results show a weak correlation between these two forms of evaluation. The main drawback of the company evaluation is that it is a costly and time-consuming task. Each idea must be individually evaluated and the participation of experts related to the subject of the idea is continuously required.

Obtained results using regression analysis clearly highlight the importance of comments for the identification of innovators (Bailey and Horvitz 2010). The number of comments received and sent is two of the three significant antecedents of the condition of being an innovator. This result can be attributed to the fact that it is much more complex to post a comment than a score. The in- and out-degree analysis show that participation through the scoring systems is much higher than participation through commenting on ideas. Besides, comments always require a justification about the critic or the improvement proposed, while scoring can be done without any justification of the score. Users score thinking more on their feeling and their own necessities than on the real applicability of the proposed ideas. Another important point is that participation by commenting on other users' ideas promotes the construction of knowledge through the interactions with other users. Obtained results point out that the best ideas are those that receive more comments. This can be attributed to the social dimension of knowledge, in the sense that ideas are improved or refined through the generated debate, leading to more concrete and well-formulated ideas, easier to be implemented by the organisation. Innovators are also characterised by their involvement with the community by commenting on other users' ideas.

\section{Implications for researchers}

From a theoretical point of view, this study is aligned with von Hippel's lead user theory about lead user identification (von Hippel 1986, 1988). Several of the identified characteristics such as popularity or high involvement with the community were already anticipated in von Hippel's previous studies. Obtained results reinforce the alternative of identifying best ideas through lead users rather than using collective intelligence scoring systems.

This study also highlights the importance of 'commenting' as a way of building innovations through the interactions with other users. According to the obtained results the number of comments received is by far the main variable able to identify innovators. This result is in line with the social learning theory from Wenger (Wenger 1998) that argues that knowledge can only be built in the context in which it is generated. The social learning theory has been used as the theoretical base for the development of communities of practice, which in turn explains how virtual communities work (Toral, Martínez-Torres, and Barrero 2009). In the case of open innovation communities it is a mechanism for improving, refining and formulating ideas more accurately. The more comments an idea receives, the more chances it has to be implemented by a company.

\section{Implication for practitioners}

Understanding open innovation paradigm requires understanding the process by which shared ideas are finally implemented by the organisation. This study provides to practitioners and open innovation website managers a practical study about the main participation variables which are able to explain the ideas which have more chances to be implemented. From the organisation perspective, this information can be used to improve the idea evaluation procedures. Instead of relying on the collective judgement of the community, the identified innovator features can be used as a first filter in the task of looking for the best ideas. Through this way, the cost associated 
with the evaluation of ideas can be reduced. Second, this study also reveals that the collective scoring system could be improved if some justification of the posted score were demanded of participants. A score with an accompanying argument would encourage community users to think in detail about the shared ideas and their possibilities for a real implementation. Besides, the provided arguments can also be useful to build upon previous arguments. The rest of the users can be aware of why an idea is receiving a good or a bad score, and their posted scores can support or contradict previous ones.

\section{Limitations}

The problem of having a large proportion of zero values can result in a loss of important information and, thus, a diminished explanatory power, as it happens in our study. The fact of not having structural zeros means that a standard distribution like negative-binomial must be used instead of zero-inflated distributions that can properly account for a large proportion of zero values when they have a structural nature. This is an inherent limitation of open innovation communities, as only a small fraction of ideas are accepted by the organisation.

Another inherent limitation of the applied methodology is that SNA does not take into account the quality of posted ideas and comments, but their quantity. The problem of analysing the content of shared ideas and comments is that online open innovation systems receive thousands of ideas, and evaluating them one by one would be a highly time-consuming task. That is the reason why these systems rely on the judgement of the rest of the community. However, the patterns of participation have already been used to analyse the structure of communities or to characterise those users occupying relevant positions, for instance, in open source communities (Sowe, Stamelos, and Angelis 2006; Toral, Martínez-Torres, and Barrero 2010). This information could be complemented with some external information about users, as the time since they registered in the community, their persistence of participation along time, of the number of topics covered by their innovations.

Finally, another limitation is some possible bias due to the way in which shared ideas are displayed. Trending or most popular ideas are usually separately displayed and this fact may cause them to receive more comments and scores than the rest of the ideas. In general, ideas with easier accessibility will have more chances of being commented on and scored. This is the case of the IdeaStorm website, which provides a direct link to the most popular ideas.

\section{Conclusion}

This paper analyses the open innovation paradigm from the perspective of social network analysis. It models the interactions among the members of the community through their comments and scores as graphs. It has also extracted several variables based on the in- and out-degree values of users in each social network. Obtained results show that open innovation communities follow an inequality participation scheme, similar to other online communities. However, active users tend to participate in all the modalities offered by the open innovation website. Scoring systems have also been analysed. Obtained results show that the scoring system based on collective intelligence can unanimously discriminate between interesting and poor ideas. However, the best ideas according to the collective scoring system do not match with those finally implemented by the organisation. This result is explained because no previous information is provided to users about how the scoring should be done nor is any argumentation required. On the contrary, the evaluation performed by Dell is more in agreement with those ideas that have received a higher number of comments. 


\section{Notes on contributor}

M.R. Martínez-Torres is an Associate Professor in the Department of Business Administration and Marketing, University of Seville. Her research interests include evaluation of utility and use of e-learning tools, knowledge management, concept mapping and virtual communities of practice. She received her MSc degree in business administration and PhD degree in business administration, both from the University of Seville, Spain, in 1996 and 2003, respectively.

\section{References}

Antikainen, M., M. Mäkipää, and M. Ahonen. 2010. Motivating and supporting collaboration in open innovation. European Journal of Innovation Management 13, no. 1: 100-19.

Bailey, B.P., and E. Horvitz. 2010. What's your idea?: A case study of a grassroots innovation pipeline within a large software company. Proceedings of the SIGCHI conference on human factors in computing systems, CHI'10, in Atlanta, GA, USA, 2065-74.

Banerjee, P., R. Friedrich, and L. Morell. 2010. Open innovation at HP labs. Computer 43, no. 11: 88-90.

Basole, R.C. 2009. Visualization of interfirm relations in a converging mobile ecosystem. Journal of Information Technology 24: 144-59.

Berg-Jensen, M., C. Hienerth, and C. Lettl. 2010. Forecasting the attractiveness of user-generating designs via online-data. Proceeding of the 2010 Acadamy of Management Annual Meeting. Montreal, Canada.

Blohm, I., C. Riedl, J.M. Leimeister, and H. Krcmar. 2011. Idea evaluation mechanisms for collective intelligence in open innovation communities: Do traders outperform raters? Proceedings of 32nd international conference on information systems, in Shanghai, China, 1-24.

Chesbrough, H., W. Vanhaverbeke, and J. West. 2006. Open innovation: Researching a new paradigm. Oxford: Oxford University Press.

Clausen, T. 2013. External knowledge sourcing from innovation cooperation and the role of absorptive capacity: Empirical evidence from Norway and Sweden. Technology Analysis \& Strategic Management 25, no. 1: 57-70.

Clauset, A., C.R. Shalizi, and M.E.J. Newman. 2009. Power-law distributions in empirical data. SIAM Review 51: 661-703.

Di Gangi, P.M., and M. Wasko. 2009. Steal my idea! Organizational adoption of user innovations from a user innovation community: A case study of Dell IdeaStorm. Decision Support Systems 48, no. 1: 303-12.

Elmquist, M., T. Fredberg, and S. Ollila. 2009. Exploring the field of open innovation. European Journal of Innovation Management 12, no. 3: 326-45.

Faraj, S., S.L. Jarvenpaa, and A. Majchrzak. 2011. Knowledge collaboration in online communities. Journal of Organization Science 22, no. 5: 1224-39.

Galbraith, B., and R. McAdam. 2011. The promise and problem with open innovation. Technology Analysis \& Strategic Management 23, no. 1: 1-6.

Hinde, J., and C. Demetrio. 1998. Overdispersion: Models and estimation. Computational Statistics and Data Analysis 27: 151-70.

von Hippel, E. 1986. Lead users: A source of novel product concepts. Management Science 32, no. 7: 791-805.

von Hippel, E. 1988. The sources of innovation. New York: Oxford University Press.

Jang, T.Y. 2005. Count data models for trip generation. Journal of Transportation Engineering 131: 444-50.

Jeppesen, L.B., and K.R. Lakhani. 2010. Marginality and problem-solving effectiveness in broadcast search. Organization Science 21, no. 5: 1016-33.

Knock, N. 2001. Compensatory adaptation to a lean medium: An action research investigation of electronic communication in process involvement groups. IEEE Transactions on Professional Communication 44, no. 4: 267-85.

Lambert, D. 1992. Zero-inflated Poisson regression with an application to defects in manufacturing. Technometrics 34, no. 1: 1-14.

Laursen, K., and A.J. Salter. 2006. Open for innovation: The role of openness in explaining innovation performance among UK manufacturing firms. Strategic Management Journal 27, no. 2: 131-50.

Lee, A.H., K. Wang, J.A. Scott, K.K. Yau, and G.J. McLachlan. 2006. Multi-level zero-inflated Poisson regression modelling of correlated count data with excess zeros. Statistical Methods in Medical Research 15: 47-61.

Leimeister, J.M. 2010. Collective intelligence. Business \& Information Systems Engineering 52, no. 4: 239-42.

Lin, C.-L., and H.-Y. Kao. 2010. Blog popularity mining using social interconnection analysis. IEEE Internet Computing 14, no. 4: 41-9.

Mahr, D., and A. Lievens. 2012. Virtual lead user communities: Drivers of knowledge creation for innovation. Research Policy 41, no. 1: 167-77. 
Martínez-Torres, M.R. 2012. A genetic search of patterns of behaviour in OSS communities. Experts Systems with Applications 39, no. 18: 13182-92.

Martínez-Torres, M.R., C. Díaz, S.L. Toral, and F. Barrero. 2012. A text categorisation tool for open source communities based on semantic analysis. Behaviour and Information Technology, 32, no. 6: 532-44. doi:10.1080/0144929X.2010.529942.

Martínez-Torres, M.R., and C. Díaz-Fernandez. Forthcoming. Current issues and research trends on open source software communities. Technology Analysis \& Strategic Management.

Martínez-Torres, M.R., S.L. Toral, F. Barrero, and F. Cortés. 2010. The role of internet in the development of future software projects. Internet Research 20, no. 1: 72-86.

Morrison, P.D., J.H. Roberts, and D.F. Midgley. 2004. The nature of lead users and measurement of leading edge status. Research Policy 33: 351-62.

Pujol, J.M., R. Sangüesa, and J. Delgado. 2002. Extracting reputation in multi agent systems by means of social network topology. Proceedings of the first international joint conference on Autonomous agents and multiagent systems, AAMAS '02, in Bologna, Italy, 467-74.

Rencher, A.C. 2002. Methods of multivariate analysis. 2nd ed. Wiley Series in Probability and Statistics, Hoboken, NJ: John Wiley \& Sons.

Sowe, S., I. Stamelos, and L. Angelis. 2006. Identifying knowledge brokers that yield software engineering knowledge in OSS projects. Information and Software Technology 48, no. 11: 1025-33.

Toral, S.L., M.R. Martínez-Torres, and F. Barrero. 2009. Virtual communities as a resource for the development of OSS projects: The case of Linux ports to embedded processors. Behaviour and Information Technology 28, no. 5: 405-19.

Toral, S.L., M.R. Martínez-Torres, and F. Barrero. 2010. Analysis of virtual communities supporting OSS projects using social network analysis. Information and Software Technology 52, no. 3: 296-303.

Toral, S.L., M.R. Martínez Torres, and P. Di Gangi. 2011. User innovations through online communities from the perspective of social network analysis. Proc. 1st international conference on advanced collaborative networks, systems and applications (COLLA 2011), Luxemburgo, 40-5.

Urban, G.L., and E. von Hippel. 1988. Lead user analyses for the development of new industrial products. Management Science 34, no. 5: 569-82.

Wellman, B. 1983. Network analysis: Some basic principles. Sociological Theory 1: 155-200.

Wenger, E. 1998. Communities of practice: Learning, meaning, and identity. Cambridge: Cambridge University Press. 